\title{
BAF Complex Enhances Reprogramming of Adult Human Fibroblasts
}

\author{
Nishant Singhal ${ }^{1 *}$, Marcos J. Araúzo-Bravo ${ }^{2,3}$, Martina Sinn ${ }^{4}$ and Holm Zaehres ${ }^{4}$ \\ ${ }^{1}$ University of California at San Diego, Department of Neurosciences, 9500 Gilman Drive, 92093, La Jolla, USA \\ ${ }^{2}$ Biodonostia Health Research Institute, Computational Biology and Systems Biomedicine, Calle Doctor, Begiristain s/n, 20014, San Sebastian, Spain \\ IIKERBASQUE, Basque Foundation for Science, Alameda Urquijo 36-5, 48011 Bilbao, Spain \\ ${ }^{4}$ Max-Planck-Institute for Molecular Biomedicine, Department of Cellular and Developmental Biology, Röntgenstraße 20, 48149, Münster, Germany
}

\begin{abstract}
Chromatin remodeling molecules of the BAF complex, Brg1 and Baf155, as well as other chromatin remodeling molecules have been described to enhance Oct4, Sox2, Klf4 and c-Myc mediated reprogramming of mouse somatic cells into induced pluripotent stem cells (iPSCs). Brg1 maintains pluripotency of mouse embryonic stem cells (mESCs) by modulating the expression of pluripotent genes including Oct4 in synergy with LIF/STAT signaling. While mESCs depend on LIF/STAT signaling, human embryonic stem cells (hESCs) use bFGF signaling to maintain pluripotency. Interestingly, unlike mESCs, BAF complex in hESCs is composed of both BAF155 and BAF170, where BAF170 plays a role in maintaining hESCs pluripotency. In this study we describe how BRG1 and BAF155 enhance reprogramming of adult human fibroblasts. Overexpression of BAF155 does not affect pluripotency of hiPSCs as tested by global gene expression profiling as well as in vivo and in vitro assays. Additionally, these findings show that BRG1 and BAF155 expression can enhance reprogramming even in the absence of LIF/STAT signaling.
\end{abstract}

Keywords: iPSCs; Reprogramming; BAF complex; Brg1; Baf155; Chromatin remodeling; Human somatic cells

\section{Introduction}

After initial discovery of Oct4, Sox2, Klf4 and c-Myc [1], of more than 30 reprogramming combinations proposed so far, only a few have been shown to improve reprogramming efficiency in both human and mouse somatic cells. These efforts not only involved additional transcriptional factors but also involved use of chromatin remodeling molecules [2-4] as well as microRNAs [57]. In addition, several chemical modifications have been used to improve reprogramming efficiency [8,9]. Stoichiometry as well as a combination of reprogramming factors play an important role in quality, kinetics and efficiency. It has been shown that Sall4, Nanog, Esrrb and Lin28 generate high quality induced pluripotent stem cells (iPSCs) with more efficiency than other combinations of factors [10]. In most of the combinations, reprogramming factors are highly expressed in pluripotent cells. However, Oct4 and Sox 2 could also be replaced by lineage specifiers of the GATA family as well as other mesendodermal (ME) specifiers [11,12]. Replacement by ME specifiers proposed the "seasaw model" of reprogramming mechanisms. In addition, depending on reprogramming factors using a "stochastic model, a deterministic model or a combination of both as well as a biphasic model" have been proposed to understand reprogramming mechanisms [13]. It has been shown that Oct4 deficient oocytes are capable of reprogramming somatic nuclei by somatic cell nuclear transfer (SCNT) with efficiency similar to normal oocytes [14]. Hence, this suggests a distinct set of reprogramming factors used during SCNT. SCNT mediated reprogramming generates higher quality pluripotent cells with faster kinetics and no epigenetic memory [15]. Interestingly, Brg1 has been shown to be essential for reprogramming somatic nuclei using SCNT [16]. Previously, we demonstrated that components of esBAF chromatin remodeling complex Brg1 and Baf155 were able to improve OSK mediated reprogramming in the presence or absence of c-Myc [2]. Notably, Brg1 is required for zygotic genome activation at 2-cell stage onwards, underlying its role in SCNT mediated reprogramming [17]. Furthermore, modulating expression of the BAF complex significantly influences reprogramming of somatic progenitor cells [18]. These studies point out that chromatin remodeling plays an essential role in improving the kinetics and efficiency of reprogramming. After the above mentioned pioneering studies showing the role of BRG1 and BAF155 in improving reprogramming efficiency, several other chromatin remodeling molecules have been described in this contexts, such as: KDM2B (a H3K36me2 demethylase) [19], KDM6A (H3K27me3 demethylase) [20], WDR5 (a histone methyltransferase complex component) [21], EZH2 (a component of polycomb repressive complex 2) [22], and the catalytic domain of the methylcytosine dehydrogenaseTet1 [23]. When these factors were compared side by side for their ability to improve reprogramming efficiency using reprogrammable MEFs (MEFs having dox inducible OSKM cassette) in 3c (combination of AA, iAlk5 and CHIR), KDM2B was able to improve reprogramming efficiency while BAF155 (Smarcc1), even in the absence of Brg1 (Smarca4), improved reprogramming efficiency [24]. This study pointed out the important role of BAF complex components in reprogramming. It has been shown that Brg1 acts by enhanced Oct4 recruitment through favoring euchromatin structure [2]. In addition, it has also been shown that Brg1 potentiate LIF signaling [25] as well as by modulate expression levels of pluripotency genes including Oct4 [26,27]. Interestingly, human embryonic stem cells (hESCs) are similar to epiblast stem cells in terms of morphology and culture conditions. Unlike mESCs, bFGF is used to main pluripotency of hESCs as well as during human somatic cell reprogramming. It has been shown that culture conditions during reprogramming process can lead to alternate fate of mouse somatic cells [28]. Additionally, BAF complex in hESCs differs from the mouse esBAF in that it also contains BAF170 (SMARCC2) in addition to

*Corresponding author: Nishant Singhal, University of California at San Diego Department of Neurosciences, 9500 Gilman Drive, 92093, La Jolla, USA, Tel: 858822-3669; E-mail: nisinghal@ucsd.edu

Received March 21, 2016; Accepted April 13, 2016; Published April 20, 2016

Citation: Singhal N, Araúzo-Bravo MJ, Sinn M, Zaehres H (2016) BAF Complex Enhances Reprogramming of Adult Human Fibroblasts. J Stem Cell Res Ther 6: 336. doi:10.4172/2157-7633.1000336

Copyright: ( 2016 Singhal N, et al. This is an open-access article distributed under the terms of the Creative Commons Attribution License, which permits unrestricted use, distribution, and reproduction in any medium, provided the original author and source are credited. 
BAF155 (SMARCC1) [29]. Intrigued by these differences, we tested the role of BRG1 and BAF155 in improving reprogramming efficiency of human fibroblasts when used in combination with OSKM. BRG1 and BAF155 were found to improve the efficiency of OSKM mediated reprogramming. Overexpression of BAF155 did not affect pluripotency of human iPSCs. These observations show that overexpression of components of BAF complex can improve reprogramming efficiency irrespective of culture conditions employed without affecting pluripotency.

\section{Results}

\section{Brg1 and Baf155 enhances Oct4, Sox2, Klf4 and generation of} Myc mediated induced pluripotent stem cells

We previously showed that Brg1 and Baf155, components of the BAF complex, enhance the efficiency of mouse induced pluripotent stem cell generation from mouse embryonic fibroblasts [2]. However, it was unknown whether the same factors can also improve the reprogramming efficiency of adult human fibroblasts. Since it has been shown recently that, contrary to the BAF complex in mouse stem cells, the BAF complex in human embryonic stem cells also contains Baf170
[29], and there might be differences in its functional conservation between mouse and human. To investigate the role of Brg1 and Baf155 in human reprogramming, we utilized a reprogramming cassette consisting of optimized human Oct4, Sox2, Klf4 and c-Myc along with a tomato reporter gene (SF hOSKM-idTomato) [30]. 100,000 F134 cells were infected with SF hOSKM-idTomato. Four days later 100,000 infected cells were re-transduced with Brg1 and Baf155 or control retroviruses. Overexpression of Brg1 and Baf155 resulted in a 2-fold increase in hiPSCs colonies on day 18 (Figure 1A). In the next set of experiments, F134 cells were infected either with SF hOSKM-idTomato or SF hOSKM-idTomato plus ectopic expression of Brg1 and Baf155 at the same time. Co-transductions of Brg1 and Baf155 resulted in about 3 -fold increases in hiPSC colonies on day 18 (Figure 1B). These results indicate that co-transduction of Brg1 and Baf155 is more efficient in improving four factors mediated reprogramming rather than infection at later stages. This is probably due to an increased expression level of BRG1 and BAF155 during intermediate stages of reprogramming.

Reprogramming transgene silencing is accompanied by expression of human pluripotent cell markers

To analyze the reprogramming process in live cells, we took

A
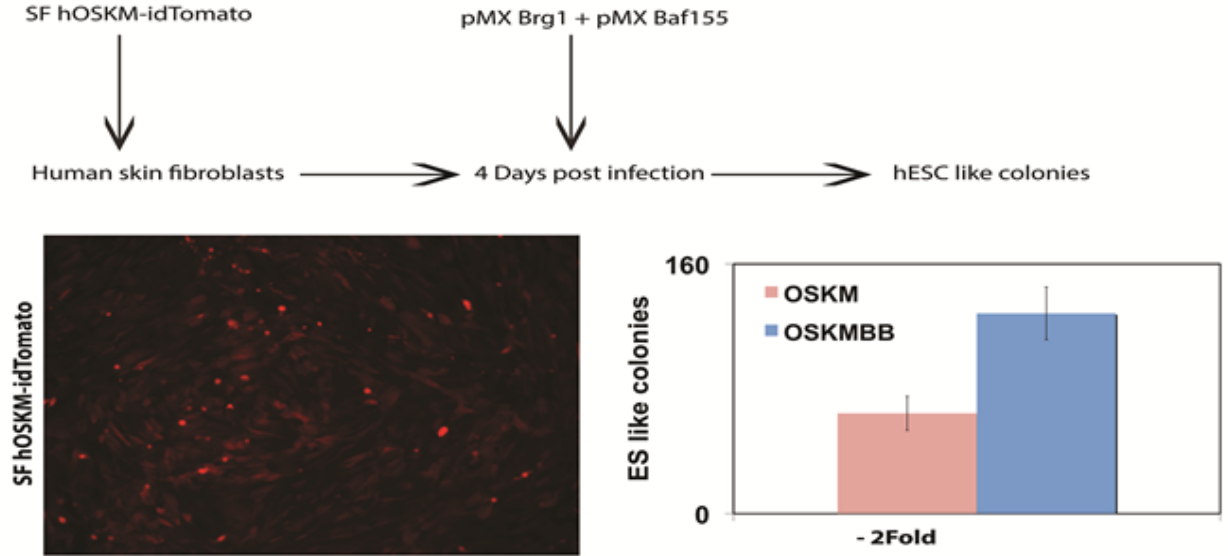

SF hOSKM-idTomato pMX Brg1 + pMX Baf155

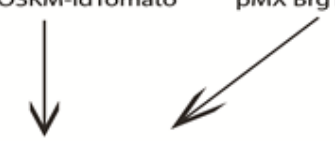

Human skin fibroblasts

hESC like colonies
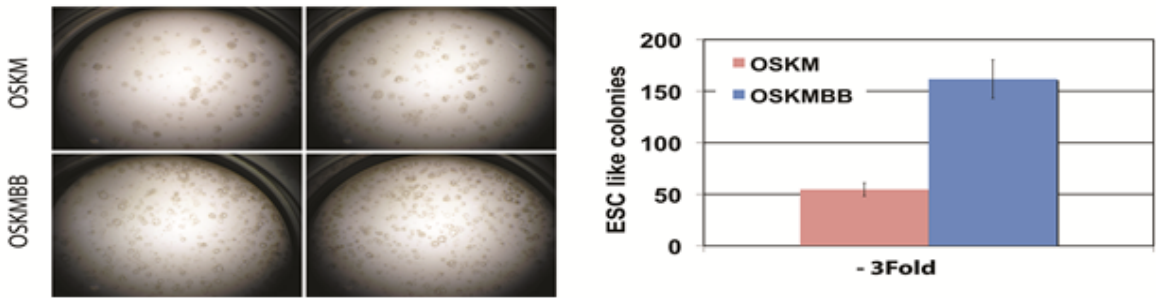

(A) Schematic representation of stepwise reprogramming scheme. Human adult fibroblasts F134 were infected with SF-OSKM-tdTomato lentivirus shown in left panel. Four days post-infection, cells were re-plated and infected with Brg1 and Baf155 viruses. Four weeks after reinfection, human stem cell-like colonies were counted. (B) Schematic representation of simultaneous reprogramming scheme. Human adult fibroblasts F134 were infected with SF-OSKM-tdTomato along with Brg1 and Baf155 simultaneously. Two examples of reprogramming have been shown in left panel. Four weeks after infection, human stem cell-like colonies were counted. Data represent mean \pm SEM of three virus-mediated transductions $(n=3)$.

Figure 1: Induction of hiPSCs using OSKM and Brg1 and Baf155. 
Citation: Singhal N, Araúzo-Bravo MJ, Sinn M, Zaehres H (2016) BAF Complex Enhances Reprogramming of Adult Human Fibroblasts. J Stem Cell Res Ther 6: 336. doi:10.4172/2157-7633.1000336

Page 3 of 8

advantage of the reporter within the SF hOSKM-idTomato reprogramming cassette. While infected F134 cells expressed red fluorescent protein after infection, the expression was silenced after the appearance of hiPSC colonies both in hOSKM and OSKM cotransduced with Brg1 and Baf155 (hOSKMBB). To test if these colonies also express pluripotency markers upon silencing of transgenes, colonies were stained with live alkaline phosphatase. As expected, colonies showing silencing of transgenes also stained positive for alkaline phosphate (Figure 2A). These results showed the silencing of transgenes upon induction of pluripotency, which is a characteristic of pluripotent stem cells.

To further identify pluripotent colonies, we performed live staining with the pluripotency marker Tra1-81 and the cell surface marker CDy1 [31]. OSKM and OSKMBB induced hiPSC colonies were found to be positive for the expression of both markers on day 18 after transduction (Figure $2 \mathrm{~B}$ ).

\section{Human OSKMBB iPSCs characterization and in vitro differentiation potential}

Human induced pluripotent stem cell colonies identified by Tra181 and CDy1 positive staining were isolated and expanded. These hOSKMBB iPSCs clones were further characterized for the expression of pluripotency markers by immunocytochemistry. HiPSC colonies were stained to test expression of alkaline phosphatase (AP), the cell surface markers Tra1-60, Tra1-81 and SSEA4 as well as the transcription factors Oct 4 and Nanog. As expected, all OSKMBB-hiPSC clones were found to be positive for expression of the pluripotency markers (Figure 3A).

Further, the in vitro developmental potential of OSKMBB-hiPSCs colonies was tested by differentiation of hiPSC derived embryoid bodies (EBs) into three germ layers. EBs were cultured in 0.5X N2B27 with $0.2 \mu \mathrm{M}$ SB43152 medium on matrigel-coated plates for 4 weeks.

A.

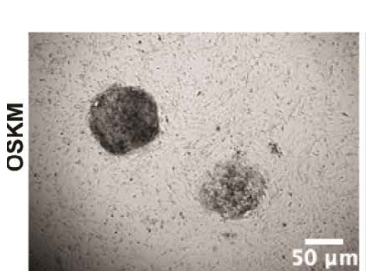

4-in-1 vector dTomato
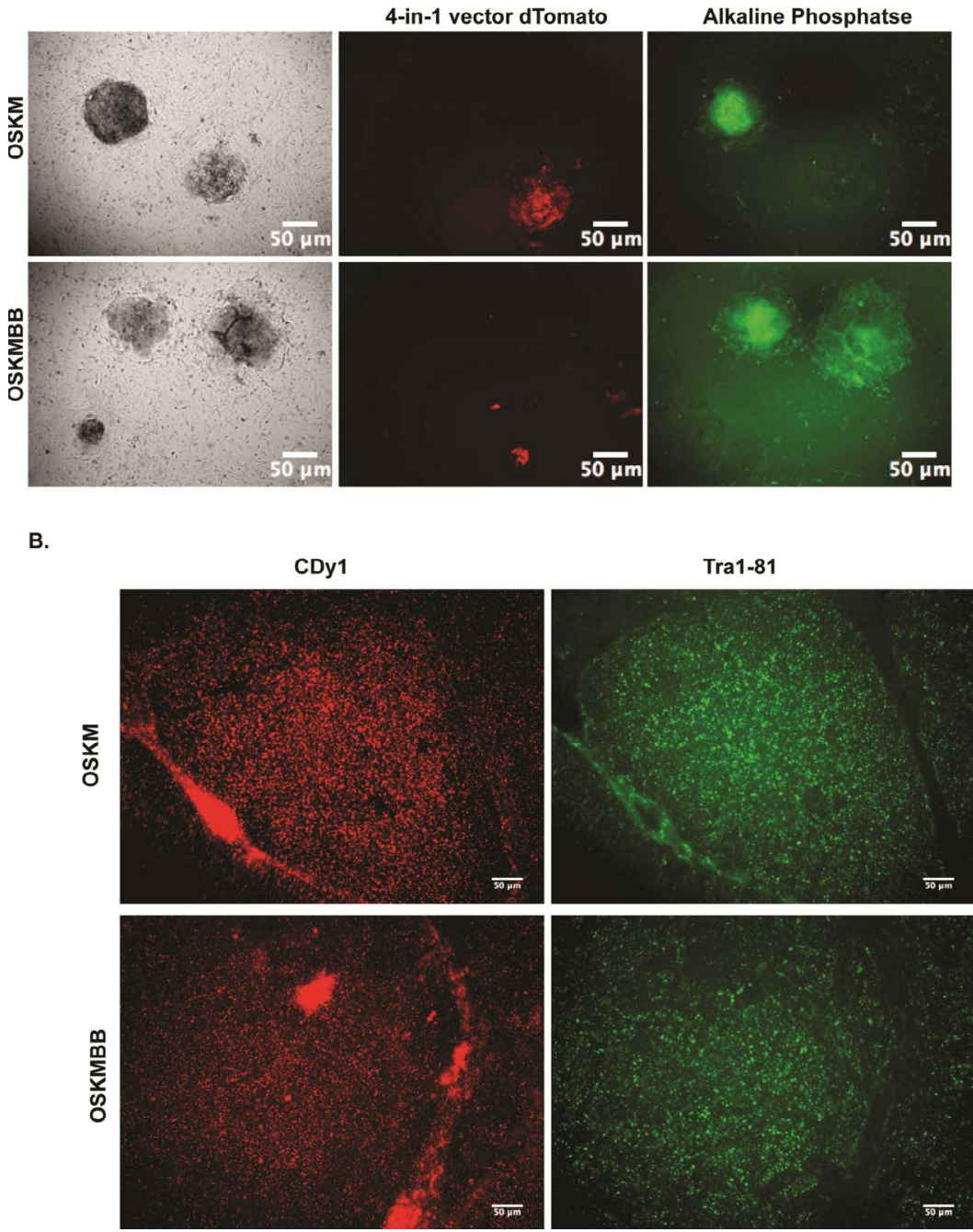

(A) Transgene silencing was observed using red fluorescent protein expression four weeks after induction of pluripotency in OSKM and OSKMBB induced reprogramming. Absence of red fluorescent expression (middle panel) in colonies with human ESCs-like morphology (left panel) demonstrates transgene silencing. These colonies also show concomitant expression of alkaline phosphatase (right panel) analyzed by live staining. (B) Live analysis of OSKM and OSKMBB hiPSC colonies during reprogramming using Tra1-81 and CDy1 show similar expression level in both types of hiPSCs.

Figure 2: Live analysis of human iPSCs. 
Citation: Singhal N, Araúzo-Bravo MJ, Sinn M, Zaehres H (2016) BAF Complex Enhances Reprogramming of Adult Human Fibroblasts. J Stem Cell Res Ther 6: 336. doi:10.4172/2157-7633.1000336

A.
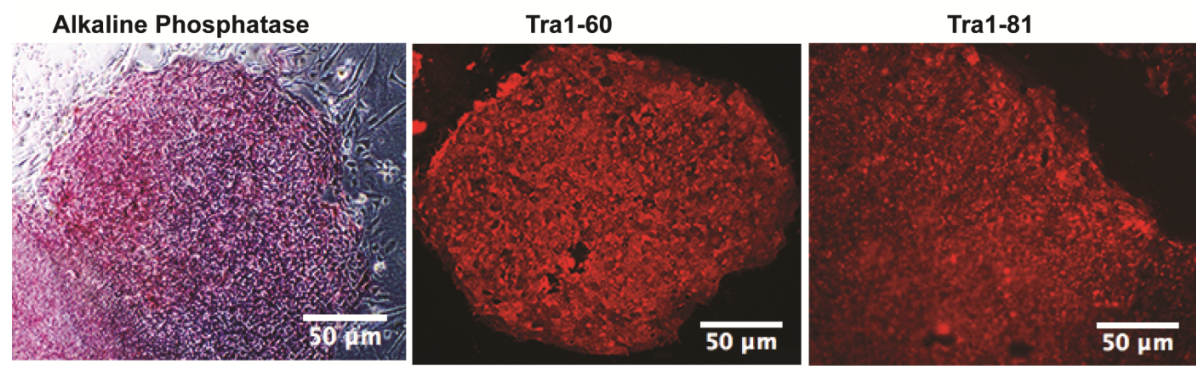

SSEA4
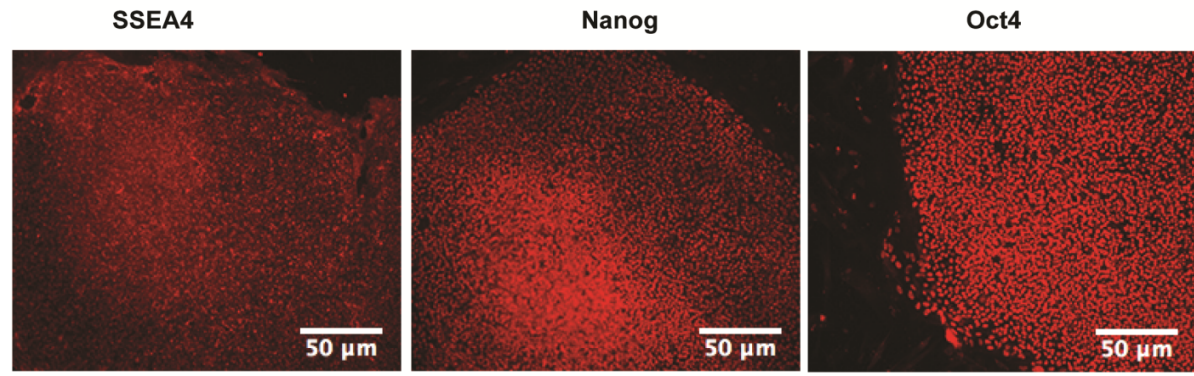

B.
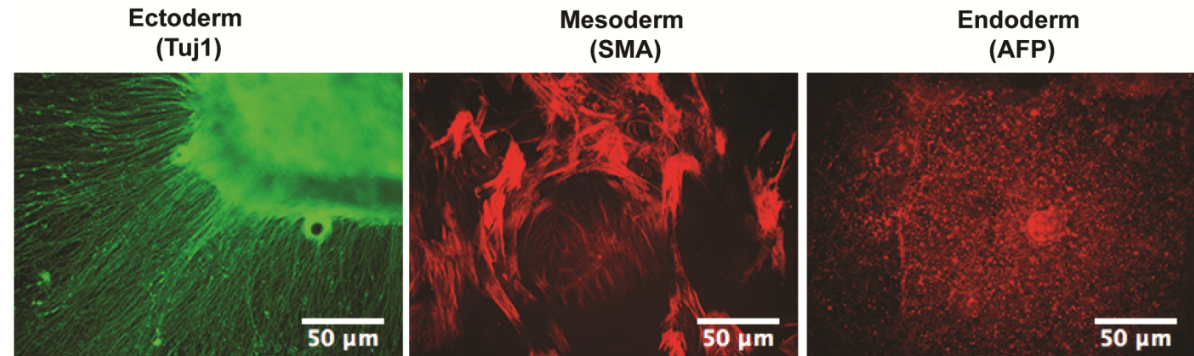

(A) Immunocytochemical analysis of pluripotency and surface markers (Alkaline phosphatase, TRA-1-60 and TRA-1-81, SSEA4, Nanog, OCT4,) in OSKMBB human iPSCs. (B) Immunofluorescence analysis of OSKMBB human iPSCs derived cells of all three germ layers after EB differentiation: ectoderm ( $\beta$-tubulin Illb; TUJ1, mesoderm ( $\alpha$-smooth muscle actin; $\alpha$-SMA) and endoderm ( $\alpha$-fetoprotein; AFP).

Figure 3: Characterization of OSKMBB hiPSCs.

These EBs efficiently differentiated into Tuj1 positive ectodermal cells. To differentiate into mesodermal and endodermal cells, EBs were cultured in the presence of FCS on gelatin coated plates for 4 weeks. Cells stained positive for the endodermal marker alpha-fetoprotein (AFP) and mesodermal marker smooth muscle actin (SMA) (Figure 3B).

These results indicated that OSKMBB-hiPSC clones have pluripotency characteristics similar to human ESCs.

In vivo differentiation potential and pluripotency gene expression profile of OSKMBB hiPSCs is similar to human embryonic stem cells

To further confirm pluripotency, we did a molecular characterization of OSKMBB hiPSCs. Quantitative RT-PCR analysis showed the expression levels of the pluripotent cell -specific transcript of OCT4, KLF4, SOX2 and NANOG in OSKMBB hiPSCs at a comparable level to both human embryonic stem cell lines $\mathrm{H} 1$ and $\mathrm{H} 9$ as well as OSKM hiPSCs (Figure 4A). Gene expression microarray profiling was carried out to further confirm the pluripotency of OSKMBB hiPSCs. Pairwise scatter plot analysis showed that global transcript profiles of OSKMBB hiPSCs have a similar expression profile to hESC line H9 as well as OSKM hiPSCs and distinguished from adult human fibroblasts. Hierarchical clustering analysis showed that OSKMBB hiPSCs cluster closely with OSKM hiPSCs and H9 as well as H1 hESCs and separated from adult human fibroblasts. Furthermore, heat map analysis showed distinct signatures in pluripotent cell lines compared to fibroblasts (Figure 4B).

Finally, teratoma analysis was performed to test in vivo differentiation potential of OSKMBB hiPSCs. Subcutaneous injection of OKMBB hiPSCs into nude mice generated teratomas. Sectioning and $\mathrm{H} \& \mathrm{E}$ analysis showed the presence of skin epithelia (ectoderm), muscles (mesoderm) and gut epithelium (endoderm) (Figure 4C).

Taken together, our analysis demonstrates that OSKMBB hiPSCs are pluripotent cells and Brg1 and Baf155 improve reprogramming of adult human fibroblasts without having any adverse effects on pluripotency. 
Citation: Singhal N, Araúzo-Bravo MJ, Sinn M, Zaehres H (2016) BAF Complex Enhances Reprogramming of Adult Human Fibroblasts. J Stem Cell Res Ther 6: 336. doi:10.4172/2157-7633.1000336

A.

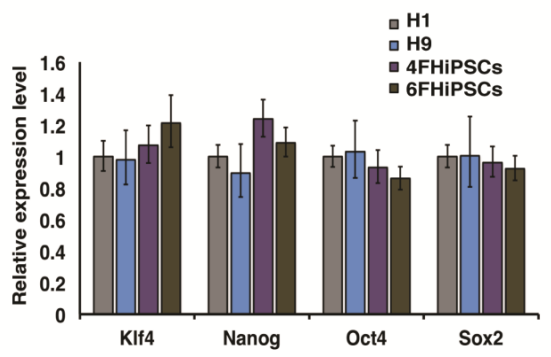

B.

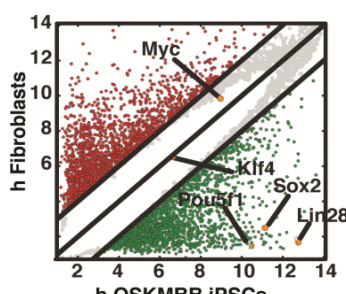

h OSKMBB iPSCs

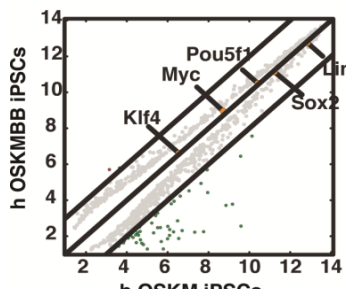

h OSKM iPSCs
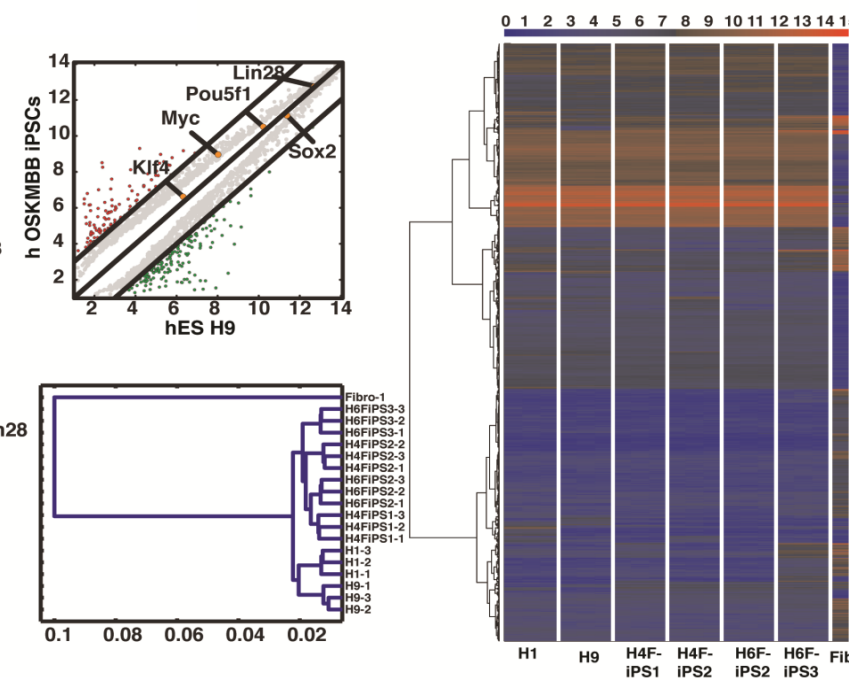

C.
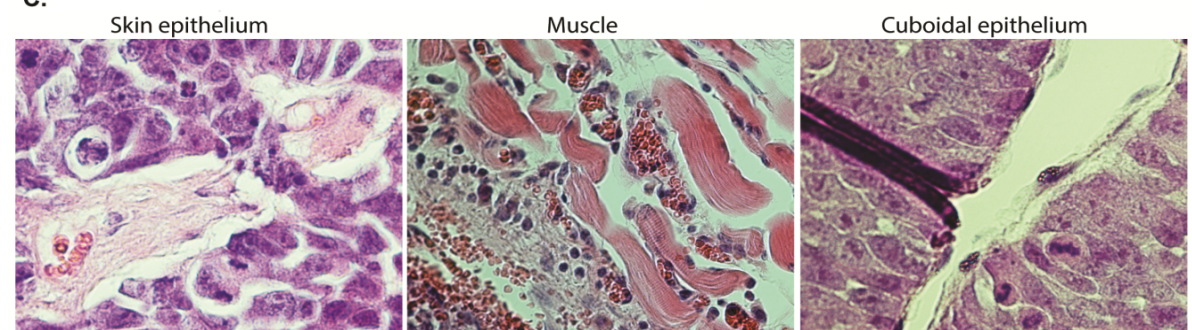

(A) Quantitative PCR analysis for pluripotent markers in H1 human ESCs, H9 human ESCs, OSKM human iPSCs and OSKMBB hiPSCs. The expression levels are relative to $\mathrm{H} 1$ human $\mathrm{ESC}$ using primers specific for endogenous transcripts, and on the logarithmic scale. Transcript levels were normalized to Gapdh expression. (B) Correlation plot analysis of human fibroblasts and OSKMBB hiPSC (upper left), OSKMBB hiPSCs and H9 (upper right) OSKMBB hiPSCs and OSKM hiPSCs (lower left). The black lines indicate twofold differences in gene expression levels between the paired cell populations. The transcript expression levels are on the log2 scale. Hierarchical cluster analysis (lower right) heat map (right panel) analysis of global gene expression from human fibroblasts, H9 human ESCs, H1 human ESCs, OSKM hiPSCs (H4FIPS) and OSKMBB hiPSCs (H6FIPS). The abscissa numbers in the hierarchical cluster correspond with the standardized Euclidean distance. A color bar (top) indicates the color code gene expression in log2 scale. (C) Teratoma formation after 6-8 weeks transplantation of OSKMBB human iPS cells into SCID mice. Teratomas were sectioned and stained with haematoxylin and eosin at 6-8weeks. Histological section of identified cells representing all three germ layers: ectoderm (skin epithelium), mesoderm (skeletal muscle) endoderm (gut epithelium).

Figure 4: Molecular and in vivo differentiation characterization.

\section{Methods}

\section{Cell culture}

Human adult fibroblast F134 cells were maintained in DMEM containing 10\% FBS (Biowest), 1X PSG, 1X NEAA, and 1X $\beta$-mercaptoethanol. 293 cells, which were used to produce retroviruses, were maintained in high-glucose DMEM (PAA) containing 10\% FBS, 1X PSG (PAA).

Human iPSCs were maintained on mitomycin-C-treated CF1 mouse feeder layers (Millipore) in human ES cell medium, which contains knockout DMEM (Invitrogen) supplemented with $20 \%$ knockout serum replacement (Invitrogen), $1 \mathrm{mM} \mathrm{l-glutamine,} \mathrm{1 \%}$ non-essential amino acids, $0.1 \mathrm{mM} \beta$-mercaptoethanol, penicillin/ streptomycin and $10 \mathrm{ng} / \mathrm{ml}$ human basic fibroblast growth factor (bFGF) (Invitrogen). iPS cells were passaged every 3 days.

\section{Induction of OSKM and OSKMBB human iPS cells}

Human adult fibroblasts F134 [32] were reprogrammed with a lentiviral vector encoding the human cDNAs of OCT4, SOX2, KLF4 and c-MYC under the control of the SFFV promoter [30]. Retroviral vectors encoding Brg1 and Baf155 [2] and the lentiviral vector were 
produced as previously described $[33,34]$. Infected fibroblasts were replated on irradiated mouse embryonic fibroblast cells in ESC medium. Approximately 25 days after transduction, iPSC colonies were selected for further expansion on the basis of their morphology.

\section{Alkaline phosphatase and immunofluorescence staining}

Live staining of human iPSCs was done using alkaline phosphatase live stain (Life technologies), Tra-1-81 (Stemgent) and CDy1 (Active motif) as per the manufacturer's protocol. Briefly, antibodies were diluted in a cell culture medium at a concentration of $2.5 \mu \mathrm{g} / \mathrm{ml}$. Used cell culture medium was aspirated and cells were incubated with an antibody containing cell culture medium for 30 minutes at $37^{\circ} \mathrm{C}$ and $5 \% \mathrm{CO}_{2}$. After incubation, cells were washed twice with a complete medium and examined under a fluorescent medium. Alkaline phosphatase staining on fixed cells was performed with the ES Cell Characterization Kit (Chemicon) according to the manufacturer's protocol. Immunofluorescence staining was performed using the following primary antibodies: AFP (Sigma, 1:100), $\alpha$-SMA (Sigma, 1:50), TUJ1 (Chemicon, 1:500), OCT4 (Santa Cruz, 1:200), Nanog (ReproCell 1:200), SSEA4 (Chemicon, 1:200), TRA-1-60 (Chemicon, 1:200) and TRA-1-81 (Chemicon, 1:200).

\section{In vitro differentiation of human iPS cells}

For immunocytochemistry, embryoid bodies (EBs) were generated from iPS cells in MEF-conditioned medium onto bacterial dishes. After 5 days, EBs were transferred into medium for three germ layers differentiation. For ectodermal differentiation: EBs was cultured in the $0.5 \mathrm{X}$ N2B27 with $0.2 \mu \mathrm{M}$ SB43152 on matrigel-coated plates for 4 weeks. For mesodermal and endodermal differentiation: DMEM medium supplemented with 15\% FBS on gelatin coated plates for 4 weeks. The medium was changed every other day.

\section{QRT-PCR}

Total RNA was extracted from cells using the RNeasy Micro Kit (QIAGEN GmbH) according to the manufacturer's instructions. cDNA synthesis was performed with the High Capacity cDNA Archive Kit (Applied Biosystems $\mathrm{GmbH}$ ) following the manufacturer's instructions with a down-scaled reaction volume of $20 \mu \mathrm{l}$. Transcript levels were determined using the ABI PRISM 7900 Sequence Detection System (Applied Biosystems) and the ready-to-use 5'-nuclease Assays-on-Demand. For real-time amplification, the template was equivalent to $5 \mathrm{ng}$ of total RNA. Polymerase chain reaction (PCR) was performed for 40 cycles for all marker genes. Oligonucleotides were designed by the TaqMan Assays-on-Demand. Assay numbers are the following: hKlf4 (Hs00358836_m1), hOct4 (Hs03005111_g1), hSox2 (Hs00602736_s1), hNanog (Hs02387400_g1). Quantification was normalized to the endogenous $\beta$ Actin gene within the log-linear phase of the amplification curve obtained for each probe/primers set using the $\Delta \Delta \mathrm{Ct}$ method (ABI PRISM 7700 Sequence Detection System, user bulletin number 2 ).

\section{Global gene expression analysis}

RNA samples for microarray analysis were prepared using RNeasy columns (Qiagen, Germany). The input in the linear amplification protocol (Ambion) was $300 \mathrm{ng}$ of total RNA per sample, with $12 \mathrm{~h}$ of in vitro transcription incorporating the biotin-labeled nucleotides. Purified and labeled cRNA was hybridized onto HumanHT-12 v4 expression BeadChips (Illumina, USA) following the manufacturer's instructions. The chips were stained with streptavidin-Cy3 (GE Healthcare) and scanned using the iScan reader (Illumina).

\section{Gene expression microarray data processing}

The bead intensities were mapped to gene information using BeadStudio 3.2 (Illumina), background correction was performed using the Affymetrix Robust Multi-array Analysis (RMA) background correction model, variance stabilization was performed using the $\log 2$ scaling, and gene expression normalization was calculated with the quantile method implemented in the lumi package of R-Bioconductor. Data post-processing and graphics were performed with in-house developed functions in Matlab. Hierarchical clusters of genes and samples were performed with a standardized Euclidean metric and the Ward's linkage method.

\section{Acceccession numbers}

The microarray data discussed have been deposited in NCBI's Gene Expression Omnibus with accession number GSE75568.

\section{Teratoma formation}

Human iPS cells $\left(3-5 \times 10^{6}\right.$ cells per mouse $)$ were injected subcutaneously into the dorsal flank of SCID mice. Teratomas were fixed in 4\% PFA overnight and embedded in paraffin 6-8 weeks after injection. Sections were stained with haematoxylin and eosin dyes.

\section{Discussion}

In this study we found that Brg1 and Baf155 significantly improve reprogramming efficiency of adult human fibroblasts. Significantly, our findings demonstrate that components of BAF complex can indeed improve reprogramming efficiency in both human and mouse cells and the mechanisms seem to be conserved. Improvement in reprogramming efficiency of adult human fibroblasts by chromatinremodeling components Brg1 and Baf155 even in absence of LIF signaling demonstrate that improved reprogramming efficiency by BAF complex is possibly mediated through chromatin remodeling, allowing better recruitment of reprogramming factors. In our previous report we found that Brg1 and Baf155 improved reprogramming efficiency of mouse somatic cells by $4-5$ fold while in human somatic cells improvement in reprogramming efficiency was about 3 fold. Mouse somatic cells are reprogrammed in the presence of LIF while human somatic cells require bFGF during reprogramming instead of using LIF. Brg1 mediated potentiation of LIF signaling to help maintain pluripotency of $\mathrm{mESCs} / \mathrm{miPSCs}[25,27]$ may provide an additive function in improving mouse somatic cells reprogramming. In this report we have shown that Brg1 and Baf155 can also improve reprogramming in the absence of LIF, showing that potentiation of LIF signaling by Brg1 and Baf155 is not essential for improvement of reprogramming efficiency. There may be several other reasons for this. For instance, while mouse iPSC colonies were selected by the Oct4 reporter expression leaving out partially reprogrammed colonies, human iPSC colonies were merely counted by morphological criteria. In addition, in this study adult human fibroblasts were used, which may have a lower propensity of reprogramming compared to embryonic fibroblasts.

In this study we observed that Brg1 and Baf155 act more efficiently when overexpressed along with OSKM rather than at a later stage. This could be due to the time it took Brg1 and Baf155 to reach critical expression levels after retroviral transduction, causing them to act inefficiently during the critical window of intermediate reprogramming when transduced at later stages. These findings are consistent with previous conclusions showing that chromatin remodeling components act at an intermediate stage of reprogramming $[2,13]$. 
Interestingly, we found that Baf155 overexpression does not affect the pluripotency of hiPSCs. It is intriguing since it was shown recently that unlike esBAF complex in mESCs, BAF complex in human embryonic stem cells contains Baf170, which is functionally important to maintain the pluripotency of hESCs [29]. This because human embryonic stem cells are more closely related to epiblast stem cells and thus represents a primed pluripotent stem cell state rather than a naïve pluripotent stem cell state. Although we did not observe any difference in pluripotency of OSKM and OSKMBB hiPSCs by global transcriptome analysis as well as in-vitro differentiation assays or teratoma assays, it's possible that OSKMBB hiPSCs may acquire Baf170 in later stages of reprogramming due to culture conditions used to maintain human iPSCs after transgene silencing. It has been shown that culture conditions can alter the fate of OSKM mediated reprogramming toward epiblast stem cells [28]. Additionally, it is also possible that OSKMBB hiPSCs exhibit naive pluripotent cells compared to OSKM hiPSCs. This will require further analysis using chimeric contribution and molecular characterization of OSKMBB hiPSCs using naïve culture conditions as described recently [35]. If this is proven, OSKMBB mediated reprogramming may provide human iPSCs of superior quality over those available through current methods.

\section{Acknowledgements}

We thank Axel Schambach for providing the lentiviral OSKM-tdTomato vector, Tobias Cantz for F134 fibroblasts; Martina Radstaak and Ingrid Gelker for human iPSCs culture; Bärbel Schäfer and Sanghamitra Singhal for histology; Toshio Kitamura for the retroviral MX vector; Hans Schöler and William C. Mobley for critical suggestions and sharing research resources; and Shelley Herron for proofreading the manuscript. The project was partly funded by the Max Planck Society for the Advancement of Sciences; Deutsche Forschungsgemeinschaft (DFG) within the Priority Program SPP 1356 "Pluripotency and Cellular Reprogramming" (grant SI 1695/1-2) and Lumind foundation.

\section{References}

1. Takahashi K, Yamanaka S (2006) Induction of pluripotent stem cells from mouse embryonic and adult fibroblast cultures by defined factors. Cell 126: 663-676. [PubMed]

2. Singhal N, Graumann J, Wu G, Araúzo-Bravo MJ, Han DW, et al. (2010) Chromatin-Remodeling Components of the BAF Complex Facilitate Reprogramming. Cell 141: 943-955. [PubMed]

3. Wang L, Du Y, Ward JM, Shimbo T, Lackford B, et al. (2014) INO80 facilitates pluripotency gene activation in embryonic stem cell self-renewal, reprogramming, and blastocyst development. Cell Stem Cell 14: 575-591. [PubMed]

4. Rais Y, Zviran A, Geula S, Gafni O, Chomsky E, et al. (2013) Deterministic direct reprogramming of somatic cells to pluripotency. Nature 502: 65-70. [PubMed]

5. Anokye-Danso F, Trivedi CM, Juhr D, Gupta M, Cui Z, et al. (2011) Highly efficient miRNA-mediated reprogramming of mouse and human somatic cells to pluripotency. Cell Stem Cell 8: 376-388. [PubMed]

6. Judson RL, Babiarz JE, Venere M, Blelloch R (2009) Embryonic stem cellspecific microRNAs promote induced pluripotency. Nat Biotechnol 27: 459-461. [PubMed]

7. Miyoshi N, Ishii H, Nagano H, Haraguchi N, Dewi DL, et al. (2011) Reprogramming of mouse and human cells to pluripotency using mature microRNAs. Cell Stem Cell 8: 633-638. [PubMed]

8. Esteban MA, Wang T, Qin B, Yang J, Qin D, et al. (2010) Vitamin C enhances the generation of mouse and human induced pluripotent stem cells. Cell Stem Cell 6: 71-79. [PubMed]

9. Martello G, Sugimoto T, Diamanti E, Joshi A, Hannah R, et al. (2012) Esrrb is a pivotal target of the Gsk3/Tcf3 axis regulating embryonic stem cell self-renewal. Cell Stem Cell 11: 491-504. [PubMed]

10. Buganim Y, Markoulaki S, van Wietmarschen N, Hoke H, Wu T, et al. (2014) The developmental potential of iPSCs is greatly influenced by reprogramming factor selection. Cell Stem Cell 15: 295-309. [PubMed]
11. Montserrat N, Nivet E, Sancho-Martinez I, Hishida T, Kumar S, et al. (2013) Reprogramming of human fibroblasts to pluripotency with lineage specifiers. Cell Stem Cell 13: 341-350. [PubMed]

12. Shu J, Wu C, Wu Y, Li Z, Shao S, et al. (2013) Induction of pluripotency in mouse somatic cells with lineage specifiers. Cell 153: 963-975. [PubMed]

13. Theunissen TW, Jaenisch R (2014) Molecular control of induced pluripotency. Cell Stem Cell 14: 720-734. [PubMed]

14. Wu G, Han D, Gong Y, Sebastiano V, Gentile L, et al. (2013) Establishment of totipotency does not depend on Oct4A. Nat Cell Biol 15: 1089-1097. [PubMed]

15. Ma H, Morey R, O'Neil RC, He Y, Daughtry B, et al. (2014) Abnormalities in human pluripotent cells due to reprogramming mechanisms. Nature 511: 177183. [PubMed]

16. Egli D, Eggan K (2010) Recipient cell nuclear factors are required for reprogramming by nuclear transfer. Development 137: 1953-1963. [PubMed]

17. Bultman SJ, Gebuhr TC, Pan H, Svoboda P, Schultz RM, et al. (2006) Maternal BRG1 regulates zygotic genome activation in the mouse. Genes Dev 20: 17441754. [PubMed]

18. Kleger A, Mahaddalkar PU, Katz SF, Lechel A, Joo JY, et al. (2012) Increased reprogramming capacity of mouse liver progenitor cells, compared with differentiated liver cells, requires the BAF complex. Gastroenterology 142: $907-$ 917. [PubMed]

19. Wang T, Chen K, Zeng X, Yang J, Wu Y, et al. (2011) The histone demethylases Jhdm1a/1b enhance somatic cell reprogramming in a vitamin-C-dependent manner. Cell Stem Cell 9: 575-587. [PubMed]

20. Mansour AA, Gafni O, Weinberger L, Zviran A, Ayyash M, et al. (2012) The H3K27 demethylase Utx regulates somatic and germ cell epigenetic reprogramming. Nature 488: 409-413. [PubMed]

21. Ang YS, Tsai SY, Lee DF, Monk J, Su J, et al. (2011) Wdr5 mediates selfrenewal and reprogramming via the embryonic stem cell core transcriptional network. Cell 145: 183-197. [PubMed]

22. Onder TT, Kara N, Cherry A, Sinha AU, Zhu N, et al. (2012) Chromatinmodifying enzymes as modulators of reprogramming. Nature 483: 598-602. [PubMed]

23. Hu X, Zhang L, Mao SQ, Li Z, Chen J, et al. (2014) Tet and TDG mediate DNA demethylation essential for mesenchymal-to-epithelial transition in somatic cell reprogramming. Cell Stem Cell 14: 512-522. [PubMed]

24. Vidal SE, Amlani B, Chen T, Tsirigos A, Stadtfeld M (2014) Combinatoria modulation of signaling pathways reveals cell-type-specific requirements for highly efficient and synchronous iPSC reprogramming. Stem cell reports 3 : 574-584. [PubMed]

25. Ho L, Miller EL, Ronan JL, Ho WQ, Jothi R, et al. (2011) esBAF facilitates pluripotency by conditioning the genome for LIF/STAT3 signalling and by regulating polycomb function. Nat Cell Biol 13: 903-913. [PubMed]

26. Ho L, Ronan JL, Wu J, Staahl BT, Chen L, et al. (2009) An embryonic stem cell chromatin remodeling complex, esBAF, is essential for embryonic stem cell self-renewal and pluripotency. Proc Natl Acad Sci U S A 106: 5181-5186. [PubMed]

27. Singhal N, Esch D, Stehling M, Schöler HR (2014) BRG1 Is Required to Maintain Pluripotency of Murine Embryonic Stem Cells. Biores Open Access 3: 1-8. [PubMed]

28. Han DW, Greber B, Wu G, Tapia N, Araúzo-Bravo MJ, et al. (2011)Direct reprogramming of fibroblasts into epiblast stem cells. Nat Cell Biol 13: 66-71. [PubMed]

29. Zhang X, Li B, Li W, Ma L, Zheng D, et al. (2014) Transcriptional repression by the BRG1-SWI/SNF complex affects the pluripotency of human embryonic stem cells. Stem cell reports 3: 460-474. [PubMed]

30. Warlich E, Kuehle J, Cantz T, Brugman MH, Maetzig T, et al. (2011) Lentiviral vector design and imaging approaches to visualize the early stages of cellular reprogramming. Mol Ther 19: 782-789. [PubMed]

31. Kang NY, Yun SW, Ha HH, Park SJ, Chang YT (2011) Embryonic and induced pluripotent stem cell staining and sorting with the live-cell fluorescence imaging probe CDy1. Nat Protoc 6: 1044-1052. [PubMed]

32. Sgodda M, Mobus S, Hoepfner J, Sharma AD, Schambach A, et al. (2013) Improved hepatic differentiation strategies for human induced pluripotent stem cells. Curr Mol Med 13: 842-855. [PubMed] 
Citation: Singhal N, Araúzo-Bravo MJ, Sinn M, Zaehres H (2016) BAF Complex Enhances Reprogramming of Adult Human Fibroblasts. J Stem Cell Res Ther 6: 336. doi:10.4172/2157-7633.1000336

Page 8 of 8

33. Kim JB, Greber B, Araúzo-Bravo MJ, Meyer J, Park KI, et al. (2009) Direct reprogramming of human neural stem cells by OCT4. Nature 461: 649-643. [PubMed]

34. Zaehres H, Kögler G, Arauzo-Bravo MJ, Bleidissel M, Santourlidis S, et al.
(2010) Induction of pluripotency in human cord blood unrestricted somatic stem cells. Experimental hematology 38: 809-818. [PubMed]

35. Hanna J, Cheng AW, Saha K, Kim J, Lengner CJ, et al. (2010) Human embryonic stem cells with biological and epigenetic characteristics similar to those of mouse ESCs. Proc Natl Acad Sci U S A 107: 9222-9227. [PubMed] 